\title{
Identification of a human synaptotagmin-1 mutation that perturbs synaptic vesicle cycling
}

\author{
Kate Baker, ${ }^{1}$ Sarah L. Gordon, ${ }^{2}$ Detelina Grozeva, ${ }^{1}$ Margriet van Kogelenberg, ${ }^{3}$ Nicola Y. Roberts, ${ }^{1}$ Michael Pike, ${ }^{4}$ Edward Blair, ${ }^{5}$ \\ Matthew E. Hurles, ${ }^{3}$ W. Kling Chong, ${ }^{6}$ Torsten Baldeweg, ${ }^{7}$ Manju A. Kurian, ${ }^{7,8}$ Stewart G. Boyd, ${ }^{7}$ UK10K Consortium, ${ }^{9}$ \\ Michael A. Cousin, ${ }^{2}$ and F. Lucy Raymond ${ }^{1}$ \\ 'Department of Medical Genetics, Cambridge Institute for Medical Research, University of Cambridge, Wellcome Trust/MRC Building, Cambridge, United Kingdom. ${ }^{2}$ Centre for Integrative Physiology, \\ University of Edinburgh, School of Biomedical Sciences, Edinburgh, United Kingdom. ${ }^{3}$ Wellcome Trust Sanger Institute, Hinxton, Cambridgeshire, United Kingdom. ${ }^{4}$ Department of Paediatric Neurology, \\ Children's Hospital, Oxford University Hospitals NHS Trust, Oxford, United Kingdom. 'Department of Clinical Cenetics, Churchill Hospital, Oxford University Hospitals NHS Trust, Headington, \\ Oxford, United Kingdom. 'Department of Radiology, Great Ormond Street Hospital for Children NHS Foundation Trust, London, United Kingdom. ${ }^{7}$ Developmental Neuroscience Programme, \\ UCL Institute of Child Health, London, United Kingdom. ${ }^{8}$ Department of Neurology, Great Ormond Street Hospital for Children NHS Foundation Trust, London, United Kingdom. \\ ${ }^{9}$ The collaborative group is detailed in supplemental material.
}

\begin{abstract}
Synaptotagmin-1 (SYT1) is a calcium-binding synaptic vesicle protein that is required for both exocytosis and endocytosis. Here, we describe a human condition associated with a rare variant in SYT1. The individual harboring this variant presented with an early onset dyskinetic movement disorder, severe motor delay, and profound cognitive impairment. Structural MRI was normal, but EEC showed extensive neurophysiological disturbances that included the unusual features of low-frequency oscillatory bursts and enhanced paired-pulse depression of visual evoked potentials. Trio analysis of whole-exome sequence identified a de novo SYT1 missense variant (I368T). Expression of rat SYT1 containing the equivalent human variant in WT mouse primary hippocampal cultures revealed that the mutant form of SYT1 correctly localizes to nerve terminals and is expressed at levels that are approximately equal to levels of endogenous WT protein. The presence of the mutant SYT1 slowed synaptic vesicle fusion kinetics, a finding that agrees with the previously demonstrated role for I368 in calciumdependent membrane penetration. Expression of the I368T variant also altered the kinetics of synaptic vesicle endocytosis. Together, the clinical features, electrophysiological phenotype, and in vitro neuronal phenotype associated with this dominant negative SYT1 mutation highlight presynaptic mechanisms that mediate human motor control and cognitive development.
\end{abstract}

\section{Introduction}

Many postsynaptic genes and processes have been linked to neurodevelopmental disorders (1), and it is increasingly apparent that presynaptic dysfunction is another major contributory pathway. Rare variants have been identified in genes that regulate presynaptic processes that ultimately affect neurotransmitter release (2). However, the final effector mechanisms that couple neuronal activity to synaptic vesicle (SV) fusion at central synapses have not yet been implicated in the pathophysiology of any neurodevelopmental disorder.

The synaptotagmins are a family of integral SV proteins required for the coupling of activity-dependent calcium influx to SV fusion at central synapses (3). Synaptotagmin-1 (SYT1) is the calcium sensor for fast synchronous evoked neurotransmitter release, since its deletion results in the specific ablation of this event (4, 5). SYT1 triggers SV fusion first by binding calcium via highly conserved cytoplasmic C2A and C2B domains, followed by penetration of the plasma membrane bilayer by a series of hydrophobic residues within these domains (6-8). This event may either destabilize the plasma membrane, lowering the energy barrier for SV

Conflict of interest: The authors have declared that no conflict of interest exists Submitted: October 29, 2014; Accepted: January 8, 2015.

Reference information: J Clin Invest. 2015;125(4):1670-1678. doi:10.1172/JCI79765. fusion, or act as an electrostatic switch, bringing the SV and target membranes in close apposition to drive their fusion (9). Ablation of SYT1 expression also affects SV endocytosis, resulting in a slowing of retrieval kinetics (10-13). The calcium-binding ability of SYT1 may also be required to accelerate SV retrieval, since experimental mutagenesis of specific residues that coordinate calcium binding did not rescue SV retrieval kinetics when expressed in SYT1-knockout neurons (14). Thus SYT1 exerts a complex influence on synaptic transmission, with both fundamental and facilitatory roles in neurotransmitter release and SV endocytosis, respectively.

Despite the key role for SYT1 in presynaptic function, there has been no confirmation to date that this gene and the molecular events it controls are essential for human neurodevelopment, because no individual with a pathogenic SYT1 variant (Synaptotagmin-1, OMIM ${ }^{\star} 185605$ ) has been identified. However, likely pathogenic variants have been reported in SYT14 and SYT2, associated with spinocerebellar ataxia and peripheral neuropathy, respectively, suggesting that impaired synaptotagmin function can be pathogenic, although via mechanisms that are likely to differ between isoforms $(15,16)$. We report a case of a human neurodevelopmental disorder associated with a rare variant in SYT1 and demonstrate that this variant exerts a dominant negative effect on SV cycling at mammalian central nerve terminals. 


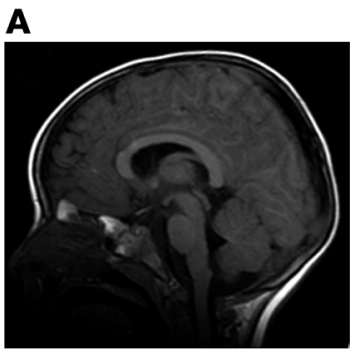

B

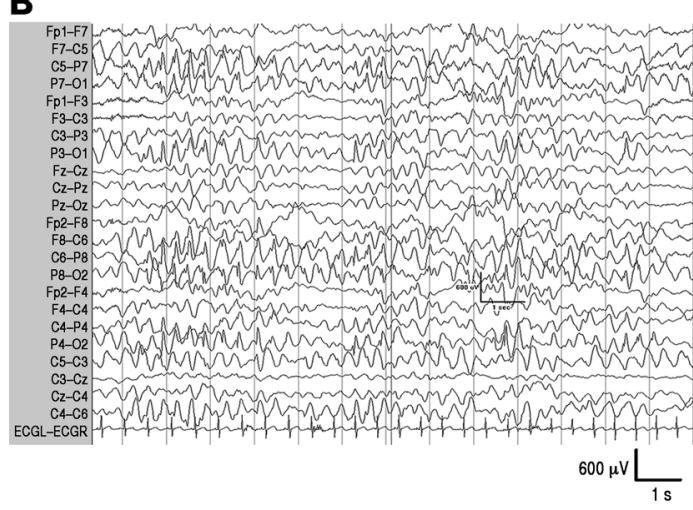

C

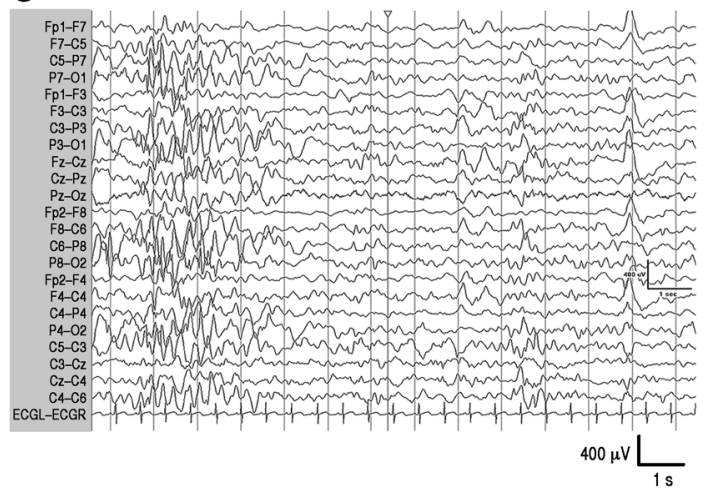

D

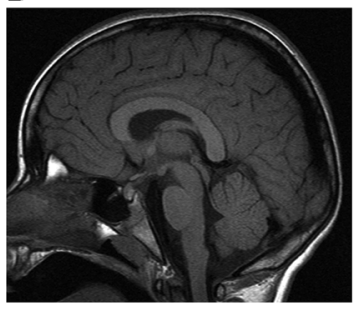

E

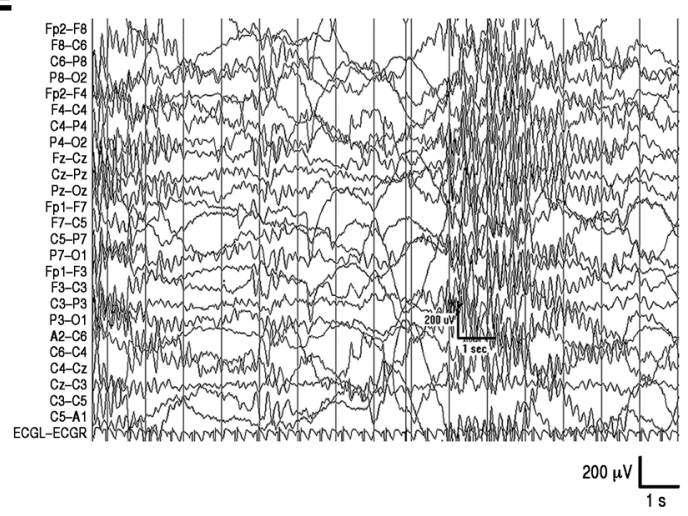

$\mathbf{F}$

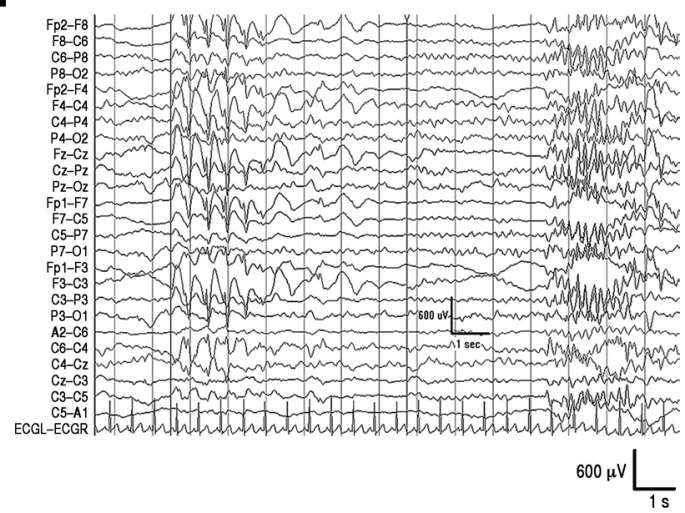

Figure 1. MRI and EEG in proband with de novo heterozygous SYT1 I368T mutation. (A) MRI at 2 years; (B) EEG at 2 years (awake); (C) EEG at 2 years (asleep); (D) MRI at 8 years; (E) EEG at 8 years (awake); (F) EEG at 8 years (asleep). T1-weighted MR images (midline sagittal slices) are presented in radiological orientation. See also Supplemental Figure 1 (spectral analysis).

\section{Results}

Clinical history. The proband is the second child of nonconsanguineous parents of European descent. There is no family history of neurological or neurodevelopmental disability. There were no medical complications of pregnancy, and he was born by spontaneous vaginal delivery at 34 weeks gestation (birth weight 50th percentile; no resuscitation required). Congenital features were bilateral talipes equinovarus (corrective surgery at 9 months) and bilateral esotropia (corrective surgery at 13 months).

During infancy, he was hypotonic and hypokinetic. By 3 years, he developed dystonic posturing of the limbs and choreoathetoid movements. By 8 years (Supplemental Video 1; supplemental material, including video legend, available online with this article; doi:10.1172/JCI79765DS1), neurological symptoms had progressed to a severe paroxysmal mixed hyperkinetic movement disorder, affecting his face, upper limbs, and lower limbs bilaterally. Chorea dominates, especially of the lower limbs. Ballismus of the lower limbs is also present. There is intermittent dystonic posturing of all affected body areas. Abnormal movements are maximal at night and worsen before and after purposeful motor activity. No seizures have been observed. Bulbar functions are intact, with no swallowing difficulties. There is no evidence for autonomic instability. Symptoms have been insensitive to trialled medications (sodium valproate, carbamazepine, chloral betaine, sedating antihistamines).

All domains of development are severely to profoundly delayed. He sat independently at 4 years of age, and at 8 years of age he could roll, pull to stand, and cruise around a playpen. At 10 years (current age), he can stand unsupported and has taken some independent steps. He sometimes reaches for objects, but has an immature grip. His visual behavior is severely immature, fixing momentarily on bright objects and not consistently following objects or faces. He turns toward voices and sounds but does not turn to his own name. He shows no evidence of verbal comprehension, uses no speech or communicative gesture, does not follow single component instructions, and shows no evidence of recognition memory. Eye contact is absent, and he does not imitate actions or vocalizations. When agitated, he chews and bites his hands. He appears to be aware of pain. There has been no developmental regression.

Vineland Adaptive Behaviour Scales II, survey interview form (17) was completed with a parent. At chronological age 8 years 3 months, developmental age-equivalent attainments were as follows: gross motor skills, 8 months; fine motor skills, 6 months; communication, daily living skills, and socialization, all less than 1 month.

Weight and height remain on the 50th percentile. Head circumference at 2 years 9 months was $49.5 \mathrm{~cm}$ (9th to 25 th percentile) and at 8 years 11 months was $53 \mathrm{~cm}$ (25th percentile) (UK cross-sectional reference data, 1996; ref. 18). He is not dysmorphic and has no neurocutaneous or musculoskeletal abnormalities. Cardiac, respiratory, and abdominal examinations are normal. Neurological examination reveals no tremor or fasciculation, no facial asymmetry, and normal eye movements. Drooling is present but not severe. Axial tone is reduced. Peripheral tone is normal throughout all 4 limbs, with no 

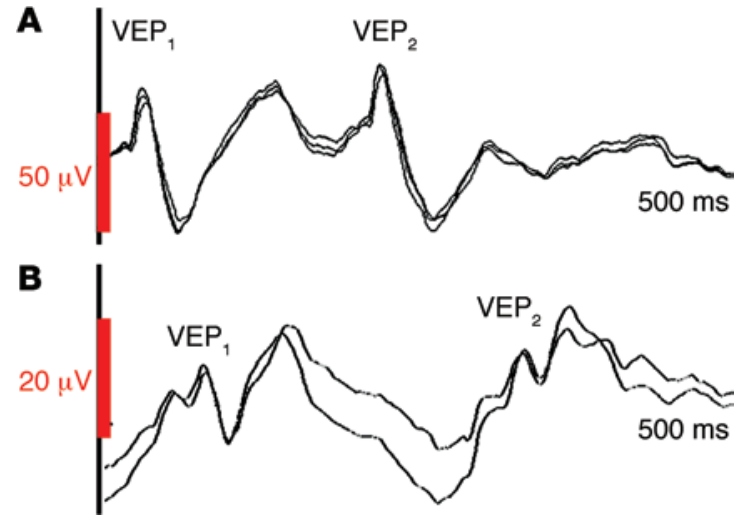

ankle clonus. Power in all 4 limbs is normal and symmetrical. Deep tendon reflexes are easily elicited in the upper limbs and brisk in the lower limbs. Plantar responses are flexor. Optic fundoscopy is normal.

In summary, the main features of this childhood-onset disorder are involuntary movements, including dystonia and chorea, accompanied by severely delayed motor development and profound intellectual disability, with no evidence of overt seizures, autonomic dysfunction, bulbar dysfunction, or developmental regression. There was no apparent in utero, perinatal, or postnatal injury or infection to suggest an acquired cause. Clinical investigations including extensive metabolic testing and chromosome analysis at $500 \mathrm{~kb}$ resolution were normal (Supplemental Methods). This severe and unexplained phenotype is not typical of any known congenital syndrome.

MRI revealed no developmental or degenerative structural brain abnormality. MRI of the brain was obtained using standard clinical acquisition sequences at $1.5 \mathrm{~T}$ at ages 2 years 2 months and 8 years 11 months. No neuroradiological abnormalities were detected, and maturation was within normal limits for chronological age at both time points (Figure 1). Magnetic resonance spectroscopy at age 8 years was within normal limits.

EEG demonstrates marked electrophysiological disturbance. Clinical EEG recordings were carried out in view of unexplained, progressive neurological symptoms at ages 2 years and 8 years (Figure 1). At both ages, background activity was diffusely slow and of high amplitude. At 2 years, the record was dominated by long periods (10 seconds to 1 minute) of very high-amplitude (up to 600 $\mu \mathrm{V})$ notched slow waves $(2-3 \mathrm{~Hz})$. During drowsiness, abnormal discharges were almost continuous, and midcentral 5-6 Hz spike activity appeared. Sleep was characterized by intermittent showers of high-amplitude, notched slow-wave activity $(0.5-4 \mathrm{~Hz})$ originating posteriorly with variable spread. At 8 years, abnormal activity had evolved into bursts of low-frequency (5-6 Hz), high-amplitude oscillations, present during wakefulness and persistent into sleep, maximal over anterior regions. These low-frequency oscillatory bursts were not linked to external stimulation and not simultaneous with motoric abnormalities or altered behavior. Occasional 2- to 3-second runs of high-amplitude spike-wave complexes were observed over frontocentral regions during sleep only.

Global electrophysiological properties were quantified via spectral analysis at age 8 years and compared with age-matched and infant controls (Supplemental Figure 1). The proband showed increased global power $\left(205.6 \mu \mathrm{V}^{2}\right.$ compared with 34 to $40 \mu \mathrm{V}^{2}$ for
Figure 2. VEPs in proband with de novo heterozygous SYT1 I368T mutation and age-matched control. Paired-pulse flash stimulation at IPI of 200 ms, sweep length 500 ms. (A) Healthy 8-year-old child: amplitude of $\mathrm{VEP}_{2}$ is at least $80 \%$ of the amplitude of $\mathrm{VEP}_{1^{*}}$ (B) Proband: amplitude of $\mathrm{VEP}_{2}$ waveform is around $50 \%$ of the amplitude of $\mathrm{VEP}$. Note difference in VEP ${ }_{1}$ amplitude and latency between the proband and control. See also Supplemental Figure 2 (auditory evoked potentials).

age-matched controls and $41-68 \mu \mathrm{V}^{2}$ for infant controls), elevated relative power within the theta range as compared with controls, and reduced relative power in all other frequency bands, with a shift in theta band distribution toward posterior electrodes.

In summary, clinical EEG recordings point toward extensive disturbance to cerebral electrical activity with developmental evolution of abnormal features, no localized cortical focus, and no features diagnostic of a recognized electroclinical syndrome.

Evoked potentials highlight unusual abnormalities of cortical processing. Visual evoked potentials (VEPs) were recorded to assess basic properties of cortical excitability in a noninvasive manner not requiring subject cooperation (Figure 2). The proband's VEPs to single-pulse flash stimulation (awake, alert) were consistently reproducible and within normal limits for morphology of the N70 and P100 components, but with reduced amplitude and increased latency in comparison with a healthy age-matched control. Paired-pulse stimulation revealed enhanced depression of the VEP response to the second-flash stimulus, sensitive to interpulse interval (IPI). At IPI of 500 ms, the proband's N70/P100 amplitude to second flash was $80 \%$ to $85 \%$ of the amplitude to first flash. At IPI of $200 \mathrm{~ms}$, the amplitude of the P100 was reduced to $45 \%$ to $50 \%$ of the amplitude to first flash, and at IPI of 100 ms, second VEP amplitude was further reduced to $33 \%$ to $40 \%$. In a healthy age-matched control child, P100 amplitude elicited by second flash showed no decrement at any IPI, consistent with adult data indicating that paired-pulse depression is not normally apparent until an IPI of less than $150 \mathrm{~ms}$ (19) and developmental data indicating that VEP responses during rapid stimulation are normally mature by age 3 years (20).

Auditory event-related potentials (AEPs) were recorded to assess the maturation of cortical information processing (Supplemental Figure 2). Comparison with a developmental data set obtained using the same recording equipment and stimulation protocol indicated that the morphology and latency of the proband's AEPs at age 8 years were similar to those of healthy infants aged 9 to 14 months (21). In contrast, age-appropriate AEPs were observed in children with epilepsy and intellectual disability due to known structural pathology, indicating that infant-like cortical information processing observed in the proband is not typical of severe neurodevelopmental disorder.

Identification of SYT1 1368 T variant. Whole-exome sequencing was performed on DNA from the proband and both parents within the UK10K project (Rare Diseases Sample Sets, www.uk10k.org). 
A
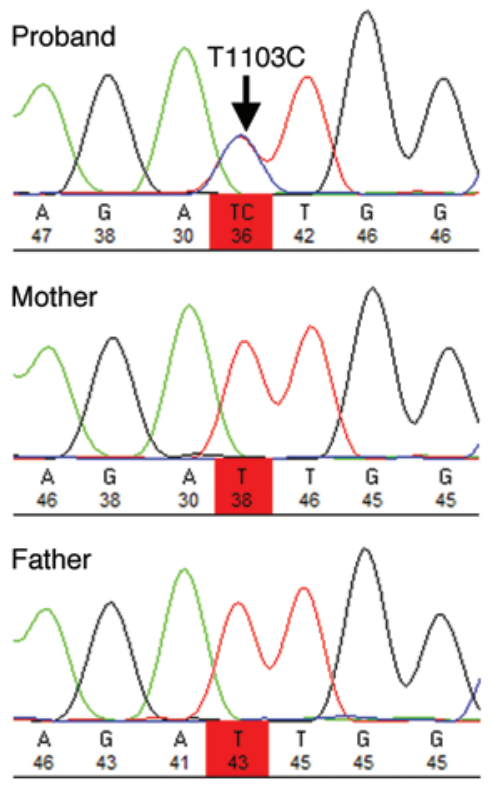

B

C. elegans
G. gallus
M. musculus
R. norvegicus
C. Iupus
M. mulatta
B. taurus
H. sapiens
P. troglodytes
D. melanogaster
A. gambiae

C. elegans

R. norvegicus

C. lupus

M. mulatta

B. taurus

H. sapiens

A. gambiae

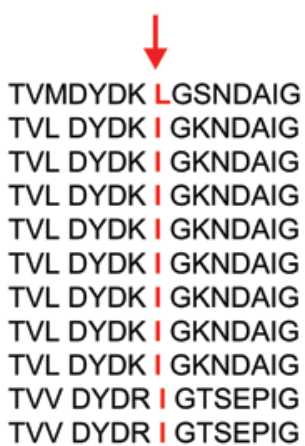

Figure 3. Identification, confirmation, and evolutionary analysis of de novo SYT1 variant. (A) Sanger sequence analysis confirming the de novo SYT1 variant. Sequence chromatograms demonstrate the presence of the variant in the proband and the reference allele in both parents. (B) Alignment of crossspecies SYT1 protein sequences, showing the position of the mutation I368T at the absolutely conserved isoleucine residue within a highly conserved region of amino acid sequence.
This included 76 probands with moderate-to-severe intellectual disability and unaffected parents. No coding variants in published genes associated with intellectual disability or congenital movement disorders were detected in the proband. Subtraction analysis to identify rare de novo variants in the proband compared with the parental DNA identified 9 variants using DeNovoGear (Supplemental Table 1). Of these variants, 6 were identified as being noncoding sequence variants (intronic, $3^{\prime}$ UTR sequence, or within a noncoding gene). Of the remaining 3 variants, 2 were identified as common polymorphisms rs649216 and rs145771593, leaving a single heterozygous de novo variant for further analysis at (chr12:79842738, hg19) within SYT1. The heterozygous de novo nonsynonymous variant that results in an isoleucine-to-threonine substitution at position 368 (I368T) was confirmed by Sanger sequencing (Figure 3 ).

The variant is absent from the 1000 Genomes data (22), more than 13,000 alleles from the Exome Variant Server (http://evs. gs.washington.edu/EVS/), and more than 122,000 alleles from the ExAC cohort (http://exac.broadinstitute.org/). This variant was unique in the UK10K cohort of $n=9464$ alleles in 4,732 patients with a range of clinical diagnoses (UK10K Neurodevelopment and Obesity sample sets). More specifically, the neurodevelopment sample set included 2,598 individuals with autism or schizophrenia. An additional cohort of 1,425 cases with intellectual disability, of which 160 cases had an associated movement disorder, were screened for this variant, and no further cases were identified. In addition, there are no reported cases in DECIPHER where SYT1 alone has been deleted or duplicated (https://decipher.sanger.ac.uk/). These data indicate that the I368T variant observed in the patient is an extremely rare mutational event.

Analysis of coding variant frequencies within human SYT1 in publicly available databases (ExAC, EVS) shows a remarkable lack of variation in this protein, as there is only a single coding polymorphism recorded at V420I within 2 amino acids of the $3^{\prime}$ end of the 422 amino acid protein. The I368T variant lies within the C2B calcium-binding region of the SYT1 protein, which is highly conserved across evolution, with isoleucine at position 368 absolutely conserved from Drosophila melanogaster to vertebrates (Figure 3).

The SYT1 I368T variant affects both SV exocytosis and endocytosis. To determine whether the de novo SYT1 variant identified in the proband alters presynaptic function, we examined the effect of expressing rat SYT1 containing the equivalent mutation (corresponding to I367T in rat) in primary cultures of WT mouse hippocampal neurons. WT SYT1 and mutant SYT1 (I368T) were tagged at their N-terminal luminal domain with pHluorin, a green fluorescent protein with enhanced $\mathrm{pH}$ sensitivity (23), to enable visualization of their intracellular localization and quantification of SV recycling. When expressed in neurons, the localization of mutant SYT1 ${ }^{1368 \mathrm{~T}}$-pHluorin was identical to that of SYT $1{ }^{\mathrm{WT}}$-pHluorin, indicating normal targeting to nerve terminals (Figure 4, A and B). This is reflected by a similar coefficient of variation value for both SYT1 $1^{\mathrm{WT}}$-pHluorin and SYT1 ${ }^{\mathrm{I368T}}$ pHluorin, which reports how punctate their distribution is along the neuronal axon (24): (SYT1 ${ }^{\mathrm{WT}}$-pHluorin 116.2 \pm 7.3 ; SYT1 ${ }^{1368 \mathrm{~T}_{-}}$ pHluorin $124.0 \pm 7.5, n=3$ for both; $P=0.50)$. Both SYT1 $1^{\text {wT }}$ pHluorin and SYT $1^{1368 \mathrm{~T}}$-pHluorin were expressed to equivalent levels, with total SYT1 expression levels increased by approximately 1.6- to 1.8-fold over background endogenous SYT1 (fold expression over endogenous: SYT $1^{\mathrm{WT}}$-pHluorin $1.60 \pm 0.14$, $n=3$; SYT1 $1^{1368 \mathrm{~T}}$-pHluorin $\left.1.77 \pm 0.18, n=4 ; P=0.53\right)$. Thus, the SYT1 $1^{\mathrm{I} 68 \mathrm{~T}}$ mutant is expressed in mouse neurons at an approximately equal proportion to that of endogenous SYT1 (mimicking the heterozygous nature of the variant in the proband).

We next determined whether expression of the SYT1 I368T mutant affected normal SV recycling, since a mutation of the equivalent isoleucine to glutamate in Drosophila resulted in a reduction in evoked neurotransmitter release (25). This was achieved by monitoring the traffic of SYT1-pHluorin. SYT1-pHluorin has little or no fluorescence when inside SVs, which have a pH of approximately 5.5 (26). Therefore exocytosis can be viewed as an increase in fluorescence and retrieval of SYT1-pHluorin from the plasma membrane 

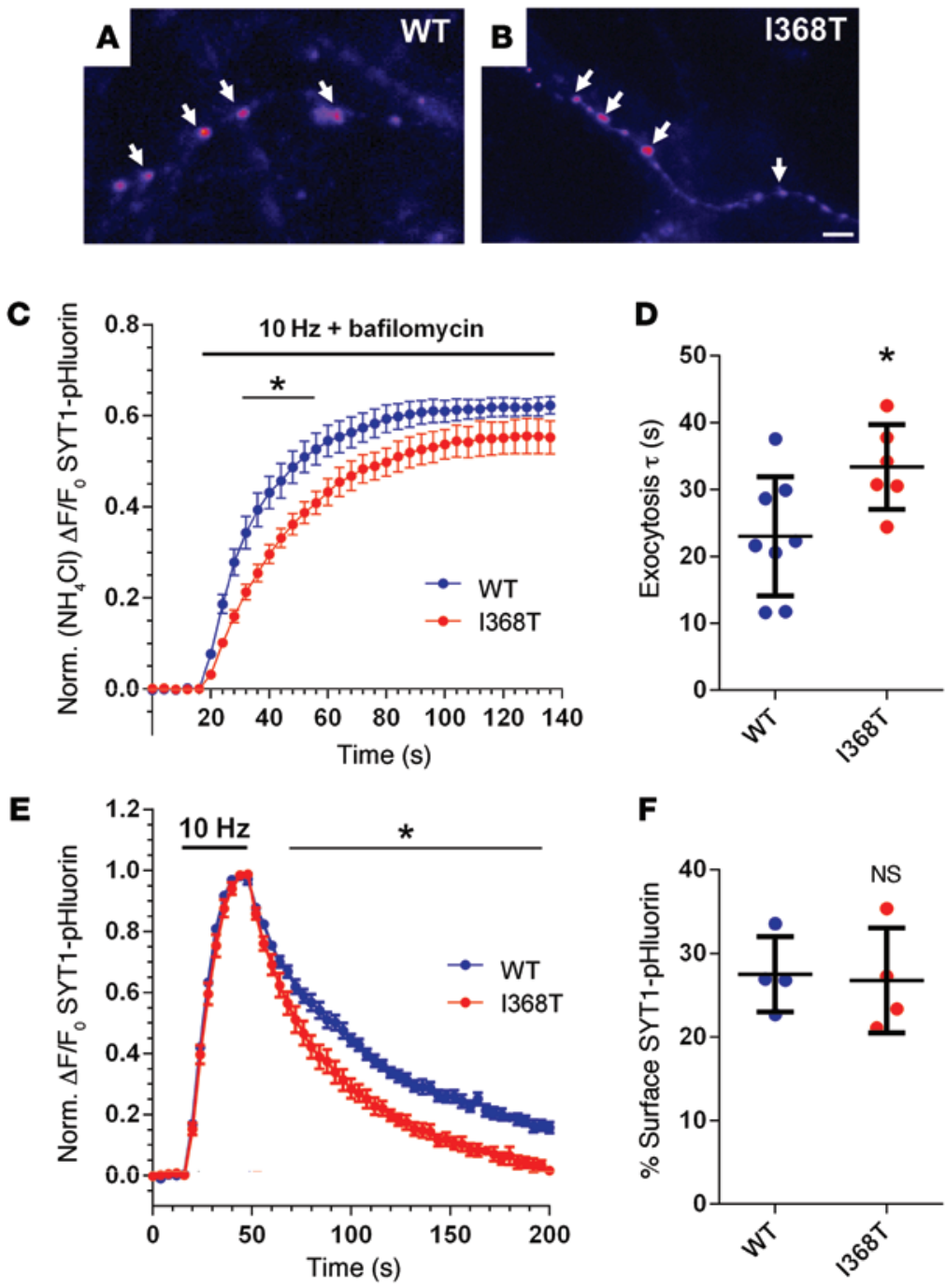

Figure 4. Effect of I368T mutation on SYT1 localization and activity-dependent trafficking. ( $\mathbf{A}$ and $\mathbf{B}$ ) Hippocampal neurons were transfected with either SYT1 ${ }^{\mathrm{WT}}$-pHluorin or SYT1 ${ }^{1368 T}$-pHluorin. Representative images show a similar punctate distribution of SYT1 ${ }^{W T}$ - pHluorin (WT, A) and SYT1 ${ }^{1368 T}$ _ pHluorin (I368T, B) along axonal segments after exposure to alkaline imaging buffer. Image is displayed in false color, with warmer colors indicating more fluorescence intensity. Arrows represent the localization of both pHluorin reporters at nerve terminals. Scale bar: $1 \mu \mathrm{m}$. (C and D) Transfected neurons were stimulated with a train of 1,200 action potentials at $10 \mathrm{~Hz}$ in the presence of $1 \mu \mathrm{M}$ bafilomycin to prevent vesicle reacidification and subsequently exposed to $\mathrm{NH}_{4} \mathrm{Cl}$ to reveal total pHluorin fluorescence ( $n=8 \mathrm{WT}, n=6$ I368T). (C) Time course of mean $\Delta \mathrm{F} / \mathrm{F}_{0}$ of SYT1-pHluorin $\pm \mathrm{SEM}$, normalized to $\mathrm{NH}_{4} \mathrm{Cl}$. ${ }^{*} P<0.05$, over time indicated (2-way ANOVA). (D) Exocytosis time constant $(\tau)$ determined by fitting a single-phase association curve to the data presented in $\mathbf{C}$. Data are presented as mean $\pm \operatorname{SEM}(n=8 \mathrm{WT}$ and $n=6 \mathrm{1368T}) .{ }^{*} P<0.03$ ( $t$ test). (E) Neurons were stimulated with a train of 300 action potentials at $10 \mathrm{~Hz}$. Graph displays the time course of mean $\Delta F / F_{0}$ of SYT1-pHluorin \pm SEM, normalized to the peak of stimulation $(n=7 \mathrm{WT}, n=6 \mathrm{I368T}) .{ }^{*} P<0.05$, over time indicated (2-way ANOVA). (F) Surface SYT1-pHluorin fluorescence, determined by normalizing SYT1-pHluorin fluorescence to $100 \%$ in alkaline buffer and $0 \%$ in acidic buffer. Data are presented as mean \pm SEM. $n=4 . P=0.86$ ( $t$ test) as a decrease (since SV endocytosis is the rate-limiting step rather than SV acidification; ref. 23). To specifically report SV exocytosis, cultures were incubated with bafilomycin A1 to block the activity of the vesicular proton pump, thereby preventing quenching of pHluorin fluorescence by SV acidification after endocytosis (27).

When cultures expressing either SYT $1^{\mathrm{WT}}$-pHluorin or SYT $1^{1368 \mathrm{~T}_{-}}$ pHluorin were challenged with a train of 1,200 action potentials in the presence of bafilomycin A1, both reporters displayed a similar peak response when normalized to the total SV pool (Figure 4C). Thus, the presence of mutant SYT1 did not affect the number of SVs that were mobilized by action potential stimulation (SYT1 ${ }^{\mathrm{WT}}$ pHluorin $62.3 \% \pm 1.9 \%, n=8$; SYT1 ${ }^{1368 \mathrm{~T}}$-pHluorin $55.2 \% \pm 3.6 \%$ of total SV pool, $n=6 ; P=0.09)$. However, the rate of SV exocytosis was greatly reduced in neurons expressing SYT1 ${ }^{1368 \mathrm{~T}}$-pHluorin compared with SYT1 ${ }^{\mathrm{WT}}$-pHluorin (Figure 4D). In summary, mutant SYT1 retards the speed of evoked SV fusion events when expressed in WT neurons.

SYT1 has a key facilitatory role in SV endocytosis; therefore, we also determined whether SYT1 ${ }^{1368 \mathrm{~T}}$-pHluorin displayed altered retrieval during endocytosis. Cultures expressing either SYT1 ${ }^{\text {WT }_{-}}$ pHluorin or SYT1 $1^{\mathrm{I} 68 \mathrm{~T}}$-pHluorin were challenged with a train of 300 action potentials delivered at $10 \mathrm{~Hz}$, and the poststimulation fluorescence decrease was monitored. SYT1 ${ }^{\mathrm{WT}}$-pHluorin fluorescence decayed with first-order kinetics as observed previously (13, $14,23)$. Intriguingly, SYT1 $1^{\mathrm{I} 68 \mathrm{~T}}$-pHluorin fluorescence returned to baseline levels with faster kinetics than SYT1 ${ }^{\mathrm{WT}}$-pHluorin (Figure $4 \mathrm{E})$, suggesting the SYT1 mutation affects its retrieval from the plasma membrane during SV endocytosis. However, this acceleration in SYT1 ${ }^{1368 T}$-pHluorin retrieval does not affect the amount of this reporter resident on the plasma membrane (Figure $4 \mathrm{~F}$ ).

To determine whether expression of the I368T mutant specifically affected SYT1 trafficking or had a general dominant negative effect on SV recycling, we examined the effect of coexpressing SYT1 fused to the cyan protein mCerulean (mCer) (28) with a separate pHluorin reporter, vGLUT-pHluorin (29). The vGLUT-pHluorin fluorescence response was observed during challenge with 1,200 action potentials in the presence of bafilomycin A1 as before. We found no significant difference in the size of the SV recycling pool between neurons expressing mCer-SYT1 ${ }^{\mathrm{WT}}$ and $\mathrm{mCer-SYT} 1^{1368 \mathrm{~T}}$ (Figure 5A, SYT1 ${ }^{\mathrm{WT}}$-pHluorin $55.3 \% \pm 2.3 \%, n=11$; SYT1 $^{1368 \mathrm{~T}}$-pHluorin $51.3 \% \pm 1.2 \%$ of total SV pool, $n=9 ; P=0.16$ ). When the rate of vGLUT-pHluorin fluorescence increase was calculated, a significant slowing was observed in $\mathrm{mCer}-\mathrm{SYT} 1^{\mathrm{I} 68 \mathrm{~T}}$-expressing neurons compared with those expressing mCer-SYT1 ${ }^{\mathrm{WT}}$ (Figure 5B). Thus, 
A

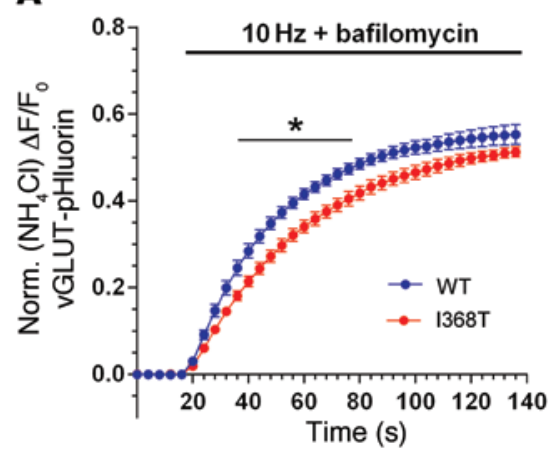

B

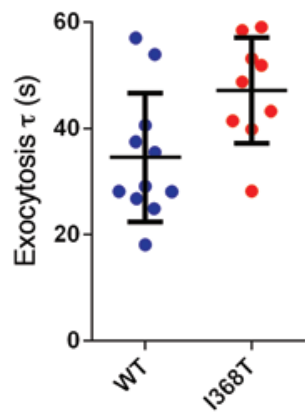

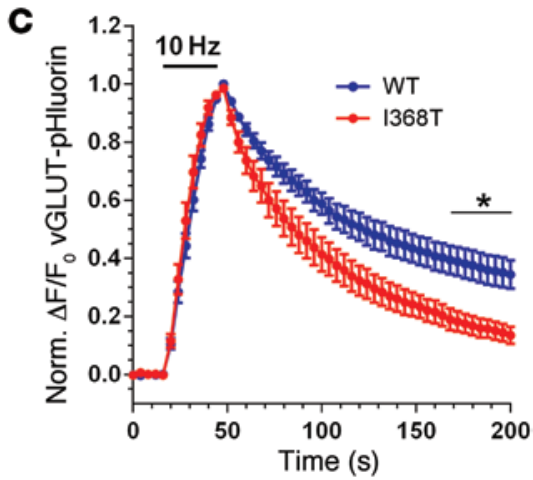

Figure 5. Effect of I368T mutation on activity-dependent SV cycling. Neurons were cotransfected with vGLUT-pHluorin and either mCer-SYT1 ${ }^{\text {WT }}$ (WT) or mCer-SYT1 $1^{1368 T}$ (I368T). (A and B) Neurons were stimulated with a train of 1,200 action potentials at $10 \mathrm{~Hz}$ in the presence of bafilomycin as described for Figure 4C. (A) Average time course of $\Delta \mathrm{F} / \mathrm{F}_{0}$ for vGLUT-pHluorin $\pm \mathrm{SEM}$, normalized to $\mathrm{NH}_{4} \mathrm{Cl}\left(n=11 \mathrm{WT}, n=9\right.$ I368T. ${ }^{*} P<0.05$, over time indicated (2-way ANOVA). (B) Average exocytosis time constant $(\tau)$ determined by fitting single-phase association curve to data presented in $\mathbf{A}$. Data are presented as mean \pm SEM ( $n=11 \mathrm{WT}, n=9 \mathrm{I368T}$ ). ${ }^{*} P<0.02$ ( $t$ test). (C) Neurons were stimulated with a train of 300 action potentials at $10 \mathrm{~Hz}$. Graph displays the average time course of $\Delta F / F_{0}$ of vGLUT-pHluorin \pm SEM, normalized to peak of stimulation $\left(n=8 \mathrm{WT}, n=9\right.$ I368T). ${ }^{*} P<0.05$, over time indicated (2-way ANOVA).

the SYT1 ${ }^{1368 \mathrm{~T}}$ mutant has a dominant negative effect on the rate of SV fusion events in mammalian central nerve terminals.

To determine whether the expression of mCer-SYT1 ${ }^{1368 \mathrm{~T}}$ influences SV endocytosis, cultures transfected with vGLUT-pHluorin and either mCer-SYT1 ${ }^{1368 \mathrm{~T}}$ or $\mathrm{mCer}-\mathrm{SYT} 1^{\mathrm{WT}}$ were stimulated with a train of 300 action potentials $(10 \mathrm{~Hz})$ in the absence of bafilomycin. Neurons expressing mCer-SYT1 ${ }^{1368 \mathrm{~T}}$ displayed a faster recovery in the vGLUT-pHluorin response when compared with mCer-SYT1 $^{\mathrm{WT}}$ (Figure $5 \mathrm{C}$ ), suggesting this mutant also displays dominant effects on global SV endocytosis kinetics.

\section{Discussion}

The identification and characterization of an extremely rare de novo variant in SYT1 associated with a severe and unusual neurodevelopmental phenotype indicate that dysregulation of rapid, synchronous SV fusion and subsequent recycling can have a profound impact on human neurological function and cognitive development. Investigation of this case exemplifies the opportunity to illuminate mechanisms of neurodevelopmental disorder when large-scale genome-sequence analysis is combined with experimental neuroscience and suggests that investigations of presynaptic mechanisms will yield further clinically relevant discoveries.

To test the hypothesis that the identified de novo nonsynonymous variant in SYT1 might explain the proband's phenotype, we first examined the properties of the gene and variant according to existing literature. Postnatal SYT1 expression is abundant throughout the cerebral cortex, whereas SYT2 is the predominant isoform in the brain stem, cranial nerves, and spinal cord (30-32), consistent with the severe cognitive impairment but normal respiratory function and neurological examination in the proband. Human transcriptome data indicate relatively low SYT1 expression in the striatum (33); however, rat in situ hybridization data point to restricted expression by dopaminergic cells in the substantia nigra pars compacta, in keeping with basal ganglia dysfunction and involuntary movement disorder (31). Human neocortical and hippocampal SYT1 expression increase during postnatal life in parallel with synaptogenesis $(33,34)$, in keeping with the evolving postnatal symptoms and severely restricted cognitive development in the proband.

To trigger SV fusion, SYT1 must bind calcium and then penetrate the lipid bilayer (6-8). The mutated isoleucine residue is highly conserved across all species and is flanked by aspartate residues that coordinate calcium binding. Furthermore, it is one of a number of hydrophobic residues within both the $\mathrm{C} 2 \mathrm{~A}$ and $\mathrm{C} 2 \mathrm{~B}$ domains that are essential for calcium-dependent membrane penetration (35). Global substitution of these hydrophobic residues with neutral amino acids inhibits calcium-dependent phospholipid binding $(7,8)$ and prevents rescue of neurotransmitter release in SYT1 KO mouse neurons (13). Conversely, substitution with more hydrophobic residues enhances both phospholipid binding and neurotransmitter release $(7,8,13,35)$. The variant residue in this study has been directly implicated in calcium-dependent membrane penetration by the $\mathrm{C} 2 \mathrm{~B}$ domain: heterozygous mutagenesis of this residue to the polar amino acid glutamate in Drosophila permitted morphologically normal brain development and SV biogenesis, but an abolition of evoked synchronous neurotransmitter release, resulting in lethal hypokinesia (25). We therefore hypothesized that the patient's heterozygous variant would have a deleterious impact on evoked, synchronous neurotransmitter release, influencing global properties of cerebral activity and affecting motor and cognitive development.

To test this prediction directly, we carried out in vitro functional studies to examine the impact of the identified heterozygous SYT1 variant on evoked SV cycling in WT mammalian neurons. This resulted in overexpression of SYT1 above endogenous levels; however, the ratio of mutant to WT SYT1 (0.8:1) approximated to that expected of heterozygous expression. Expression of the equivalent mutation in rat SYT1 (I367T) resulted in a dominant negative effect on SV fusion kinetics reported by either SYT1-pHluorin or vGLUT-pHluorin; since the same SV phenotype was observed with both reporters, we suggest that heterozygous expression of SYT1 ${ }^{1368 \mathrm{~T}}$ slows exocytosis either by limiting evoked synchronous release or via desynchronization, leading to increased asynchronous release. The dominant negative effect could result from 
the need for multiple soluble NSF attachment protein receptor (SNARE) complexes to fuse a SV; thus, the displacement of endogenous SYT1 by SYT1 ${ }^{1368 \mathrm{~T}}$ in some complexes results in inefficient fusion events (23). Alternatively, the deficiency in SV fusion could stem from multimerization of mutant SYT1 ${ }^{1368 \mathrm{~T}}$ with WT SYT1. The C2AB domains of SYT1 can oligomerize at membranes into ring-like structures in vitro that dissociate upon addition of $\mathrm{Ca}^{2+}$ (36). Formation of this structure is proposed to prevent completion of the SNARE complex assembly, with calcium removing this potential fusion clamp. Thus mutant SYT1 $1^{\mathrm{I} 68 \mathrm{~T}}$ may act in a dominant negative manner by oligomerizing with WT SYT1 and reducing the efficiency of calcium-triggered disassembly.

SYT1 is also required for efficient SV endocytosis in central nerve terminals, with homozygous knockout of SYT1 resulting in retarded SV retrieval kinetics (10-14). We observed that the retrieval of SYT1 $1^{1368 \mathrm{~T}}$ was faster than that of SYT1 $1^{\mathrm{WT}}$ and that SV endocytosis occurred more quickly with SYT1 $1^{1368 \mathrm{~T}}$ expression compared with SYT1 ${ }^{\mathrm{WT}}$. Accelerated SV endocytosis has been observed before, in neurons derived from stonin 2-knockout mice (37). In the absence of stonin 2, an endocytic adaptor protein selective for SYT1, accelerated endocytosis was accompanied by increased expression of SYT1 at the plasma membrane due to defective SYT1 retrieval (37). However, we saw no surface stranding of SYT1 ${ }^{1368 \mathrm{~T}}$, suggesting the observed acceleration of SV endocytosis is due to an alternative, currently unresolved mechanism.

The effects of the SYT1 I368T variant on evoked SV fusion and endocytosis may influence the fidelity of neurotransmission. Because of this, we evaluated the electrophysiological features of the proband to identify any abnormalities that could plausibly reflect presynaptic dysfunction. We observed reduction in the amplitude and prolonged time course of single-flash VEP, in keeping with reduced cortical excitability, but not specifically indicative of presynaptic dysfunction. A more unusual feature was enhanced frequency-dependent paired-pulse depression of the VEP. Shortterm synaptic depression is dependent on the probability of SV fusion, including replenishment of SV pools and recovery of calcium-sensitive release mechanisms, including SYT1 $(38,39)$, with presynaptic recovery predominating over postsynaptic desensitization at central mammalian synapses (40). Xu et al. (41) extensively characterized a SYT1 mouse model and identified dynamic regulatory functions for SYT1 during burst activity, showing that its knockdown impaired cortical learning via abnormal filtering of high-frequency activity. The proband's background EEG showed extensive low-frequency oscillations suggestive of impaired filtering of thalamocortical activity. Collectively, electrophysiological observations in the proband lead us to propose that abnormal SV cycling associated with this dominant negative variant disturbs the dynamic properties of synaptic transmission, severely limiting postnatal functional brain organization, as indexed by infant-like auditory evoked responses.

In summary, abnormal kinetics of neurophysiological function have been uncovered by in vivo assessment of a child with a heterozygous SYT1 mutation and in vitro investigation of the mutation in isolated mouse neurons. There is accumulating evidence that defects in the presynaptic vesicle release and reuptake cycle can contribute to the pathophysiology of severe neurodevelopmental disorders. Deletion of the SV proteins synapsin I or synap- sin II in mice results in epilepsy, learning impairments, and socialbehavioral deficits that mimic symptoms of autism (42). Cultures derived from synapsin I- or II-knockout mice have functional SV pools of reduced size, and this effect is not rescued by expression of synapsin variants identified in individuals with autism and seizure disorders $(43,44)$. Mutations in syntaxin-binding protein 1 (STXBP1; Munc-18) have been associated with epileptic encephalopathy, intellectual disability, and movement disorders (45-47), and recently mutations in syntaxin-1B (STX1B) have also been associated with temperature-sensitive seizure disorders (48), indicating that SNARE complex assembly, SV docking, and priming are important steps in the pathway leading to SV fusion (49). Mice lacking the SV protein synaptophysin (SYP) display behavioral deficits consistent with the association between human SYP mutations and intellectual disability $(50,51)$. Cultured neurons derived from SYP-deficient mice display reduced endocytic rate and SV cargo-retrieval defects $(24,52)$. Furthermore, expression of SYP mutations identified in individuals with $\mathrm{X}$-linked intellectual disability failed to rescue this cargo-retrieval defect, suggesting that disturbed kinetics of endocytosis contribute to the pathophysiology of this rare inherited neurodevelopmental disorder (53). We propose that $S Y T 1$ mutation and consequent disturbance to the SV fusion step of exocytosis and endocytosis be considered within this emerging group of presynaptic disorders, in which dynamic disruption to neurotransmission influences functional neurodevelopment, leading to a range of movement disorders and intellectual impairments.

\section{Methods}

Patient recruitment and phenotyping. Clinical phenotyping was carried out by clinical geneticists and pediatric neurologists. Neuroimaging and neurophysiology investigations at 2 time points were qualitatively reviewed by a pediatric neuroradiologist and pediatric neurophysiologist. Clinical investigations, EEG acquisition, and analysis protocols are provided in Supplemental Methods.

Exome sequencing and bioinformatics. Exome sequencing and trio de novo analysis were carried out for the proband and both parents as described in Supplemental Methods. Raw data are available at the European Genome-phenome Archive (study accession number EGAS00001000128; data set accession EGAD00001000416). The de novo SYT1 variant was confirmed by Sanger sequencing.

Functional studies. Materials for pHluorin constructs, plasmid preparation, and hippocampal neuronal culture methods are detailed in Supplemental Methods. Immunolabeling and fluorescent imaging were performed as described previously (24), and protocols are detailed in Supplemental Methods. In all cases, $n$ refers to the number of independent experiments performed.

Statistics. Statistical analyses were performed using GraphPad Prism software, and all data were tested by Student's $t$ test (2-tailed) except time-course experiments, which were assessed by 2-way ANOVA. $P<0.05$ was considered significant.

Study approval. Institutional review board approval was obtained from the Multi-Centre Research Ethics Committee for Scotland (study reference 03/0/014). The proband's parents provided written, informed consent for all aspects of the study, including publication of identifiable material. 


\section{Acknowledgments}

We acknowledge the contribution of the proband's family to this report. Clinical EEG at age 2 years was reported by Zenobia Zaiwalla (The Park Hospital for Children, Oxford, United Kingdom). EEG at age 8 years was recorded by Mark Hair (Department of Clinical Neurophysiology, Great Ormond Street Hospital). This work was supported by the Wellcome Trust (WT091310, WT098051); the Medical Research Council (G1002117); National Institute for Health Research (United
Kingdom); Action Medical Research; and the Academy of Medical Sciences/Wellcome Trust. See Supplemental Data for UK10K Consortium details.

Address correspondence to: Kate Baker, Department of Medical Genetics, Cambridge Institute for Medical Research, Cambridge Biomedical Campus, Wellcome Trust/MRC Building, Hills Road, Cambridge, CB2 OXY, United Kingdom. Phone: 44.0.1223.767812; E-mail:kb488@cam.ac.uk.
1. Zoghbi HY, Bear MF. Synaptic dysfunction in neurodevelopmental disorders associated with autism and intellectual disabilities. Cold Spring Harb Perspect Biol. 2012;4(3):a009886.

2. Waites CL, Garner CC. Presynaptic function in health and disease. Trends Neurosci. 2011;34(6):326-337.

3. Südhof TC, Rizo J. Synaptic vesicle exocytosis. Cold Spring Harb Perspect Biol. 2011;3(12):a005637.

4. Geppert M, et al. Synaptotagmin I: a major $\mathrm{Ca}^{2+}$ sensor for transmitter release at a central synapse. Cell. 1994;79(4):717-727.

5. Bacaj T, et al. Synaptotagmin-1 and synaptotagmin-7 trigger synchronous and asynchronous phases of neurotransmitter release. Neuron. 2013;80(4):947-959.

6. Bai J, Wang P, Chapman ER. C2A activates a cryptic $\mathrm{Ca}(2+)$-triggered membrane penetration activity within the C2B domain of synaptotagmin I. Proc Natl Acad Sci U S A. 2002;99(3):1665-1670.

7. Martens S, Kozlov MM, McMahon HT. How synaptotagmin promotes membrane fusion. Science. 2007;316(5828):1205-1208.

8. Hui E, Johnson CP, Yao J, Dunning FM, Chapman ER. Synaptotagmin-mediated bending of the target membrane is a critical step in $\mathrm{Ca}(2+)$ regulated fusion. Cell. 2009;138(4):709-721.

9. Jahn R, Fasshauer D. Molecular machines governing exocytosis of synaptic vesicles. Nature. 2012;490(7419):201-207.

10. Jorgensen EM, Hartwieg E, Schuske K, Nonet ML, Jin Y, Horvitz HR. Defective recycling of synaptic vesicles in synaptotagmin mutants of Caenorhabditis elegans. Nature. 1995;378(6553):196-199.

11. Poskanzer KE, Marek KW, Sweeney ST, Davis GW. Synaptotagmin I is necessary for compensatory synaptic vesicle endocytosis in vivo. Nature. 2003;426(6966):559-563.

12. Poskanzer KE, Fetter RD, Davis GW. Discrete residues in the $\mathrm{c}$ (2)b domain of synaptotagmin I independently specify endocytic rate and synaptic vesicle size. Neuron. 2006;50(1):49-62.

13. Yao J, Kwon SE, Gaffaney JD, Dunning FM, Chapman ER. Uncoupling the roles of synaptotagmin I during endo- and exocytosis of synaptic vesicles. Nat Neurosci. 2012;15(2):243-249.

14. Yao LH, et al. Synaptotagmin 1 is necessary for the $\mathrm{Ca} 2+$ dependence of clathrin-mediated endocytosis. J Neurosci. 2012;32(11):3778-3785.

15. Doi $\mathrm{H}$, et al. Exome sequencing reveals a homozygous SYT14 mutation in adult-onset, autosomal-recessive spinocerebellar ataxia with psychomotor retardation. Am J Hum Genet.

\section{1;89(2):320-327.}

16. Herrmann DN, et al. Synaptotagmin 2 mutations cause an autosomal-dominant form of lambert-eaton myasthenic syndrome and nonprogressive motor neuropathy. Am J Hum Genet. 2014;95(3):332-339.

17. Sparrow S, Cicchetti D, Balla D. Vineland Adaptive Behaviour Scales. 2nd ed. Upper Saddle River, New Jersey, USA: Pearson Education Ltd.; 2005.

18. Child Growth Foundation. Head Circumference Chart. South Shields, United Kingdom: Harlow Printing Ltd.; 1996.

19. Hoffken O, Grehl T, Dinse HR, Tegenthoff M, Bach M. Paired-pulse behavior of visually evoked potentials recorded in human visual cortex using patterned paired-pulse stimulation. Exp Brain Res. 2008;188(3):427-435.

20. Lippe S, Roy MS, Perchet C, Lassonde M. Electrophysiological markers of visuocortical development. Cereb Cortex. 2007;17(1):100-107.

21. Werner K, Fosi T, Boyd SG, Baldeweg T, Scott RC, Neville BG. Temporal lobe impairment in West syndrome: event-related potential evidence. Ann Neurol. 2015;77(1):47-57.

22. Schuler GD, et al. A gene map of the human genome. Science. 1996;274(5287):540-546.

23. Diril MK, Wienisch M, Jung N, Klingauf J, Haucke V. Stonin 2 is an AP-2-dependent endocytic sorting adaptor for synaptotagmin internalization and recycling. Dev Cell. 2006;10(2):233-244.

24. Gordon SL, Leube RE, Cousin MA. Synaptophysin is required for synaptobrevin retrieval during synaptic vesicle endocytosis. J Neurosci. 2011;31(39):14032-14036.

25. Paddock BE, et al. Membrane penetration by synaptotagmin is required for coupling calcium binding to vesicle fusion in vivo. JNeurosci. 2011;31(6):2248-2257.

26. Sankaranarayanan S, Ryan TA. Real-time measurements of vesicle-SNARE recycling in synapses of the central nervous system. Nat Cell Biol. 2000;2(4):197-204

27. Sankaranarayanan S, Ryan TA. Calcium accelerates endocytosis of vSNAREs at hippocampal synapses. Nat Neurosci. 2001;4(2):129-136.

28. Rizzo MA, Springer GH, Granada B, Piston DW. An improved cyan fluorescent protein variant useful for FRET. Nat Biotechnol. 2004;22(4):445-449.

29. Voglmaier SM, et al. Distinct endocytic pathways control the rate and extent of synaptic vesicle protein recycling. Neuron. 2006;51(1):71-84.

30. UK Brain Expression Consortium. Braineac - The Brain eQTL Almanac. Braineac Web site. http:// www.braineac.org. Accessed February 4, 2015.

31. Ullrich B, et al. Functional properties of multiple synaptotagmins in brain. Neuron. 1994;13(6):1281-1291.

32. Hawrylycz MJ, et al. An anatomically comprehensive atlas of the adult human brain transcriptome. Nature. 2012;489(7416):391-399.

33. Kang HJ, et al. Spatio-temporal transcriptome of the human brain. Nature. 2011;478(7370):483-489.

34. Allen Institute for Brain Science. BrainSpan Atlas of the Developing Human Brain. BrainSpan Web site. www.brainspan.org. Accessed February 4, 2015.

35. Rhee JS, et al. Augmenting neurotransmitter release by enhancing the apparent $\mathrm{Ca}^{2+}$ affinity of synaptotagmin 1. Proc Natl Acad Sci US A. 2005;102(51):18664-18669.

36. Wang J, et al. Calcium sensitive ring-like oligomers formed by synaptotagmin. Proc Natl Acad Sci U S A. 2014;111(38):13966-13971.

37. Kononenko NL, et al. Compromised fidelity of endocytic synaptic vesicle protein sorting in the absence of stonin 2. Proc Natl Acad Sci U S A 2013;110(6):E526-E535.

38. Hennig MH. Theoretical models of synaptic short term plasticity. Front Comput Neurosci. 2013;7:154.

39. Kavalali ET. Multiple vesicle recycling pathways in central synapses and their impact on neurotransmission. J Physiol. 2007;585(pt 3):669-679.

40. Yang H, Xu-Friedman MA. Relative roles of different mechanisms of depression at the mouse endbulb of Held. JNeurophysiol. 2008;99(5):2510-2521.

41. Xu W, Morishita W, Buckmaster PS, Pang ZP, Malenka RC, Südhof TC. Distinct neuronal coding schemes in memory revealed by selective erasure of fast synchronous synaptic transmission. Neuron. 2012;73(5):990-1001.

42. Giovedi S, Corradi A, Fassio A, Benfenati F. Involvement of synaptic genes in the pathogenesis of autism spectrum disorders: the case of synapsins. Front Pediatr. 2014;2:94.

43. Fassio A, et al. SYN1 loss-of-function mutations in autism and partial epilepsy cause impaired synaptic function. Hum Mol Genet. 2011;20(12):2297-2307.

44. Corradi A, et al. SYN2 is an autism predisposing gene: loss-of-function mutations alter synaptic vesicle cycling and axon outgrowth. Hum Mol Genet. 2014;23(1):90-103.

45. Milh M, et al. Epileptic and nonepileptic features in patients with early onset epileptic encephalopathy and STXBP1 mutations. Epilepsia. 2011;52(10):1828-1834.

46. Rauch A, et al. Range of genetic mutations associated with severe non-syndromic sporadic intellectual disability: an exome sequencing study. 
Lancet. 2012;380(9854):1674-1682.

47. Kanazawa K, Kumada S, Kato M, Saitsu H, Kurihara E, Matsumoto N. Choreo-ballistic movements in a case carrying a missense mutation in syntaxin binding protein 1 gene. Mov Disord. 2010;25(13):2265-2267.

48. Schubert J, et al. Mutations in STX1B, encoding a presynaptic protein, cause fever-associated epilepsy syndromes. Nat Genet. 2014;46(12):1327-1332.
49. Rizo J, Sudhof TC. The membrane fusion enigma: SNAREs, Sec1/Munc18 proteins, and their accomplices - guilty as charged? Annu Rev Cell Dev Biol. 2012;28:279-308.

50. Tarpey PS, et al. A systematic, large-scale resequencing screen of $\mathrm{X}$-chromosome coding exons in mental retardation. Nat Genet. 2009;41(5):535-543.

51. Schmitt U, Tanimoto N, Seeliger M, Schaeffel F, Leube RE. Detection of behavioral alterations and learning deficits in mice lacking synaptophysin. Neuroscience. 2009;162(2):234-243.

52. Kwon SE, Chapman ER. Synaptophysin regulates the kinetics of synaptic vesicle endocytosis in central neurons. Neuron. 2011;70(5):847-854.

53. Gordon SL, Cousin MA. X-linked intellectual disability-associated mutations in synaptophysin disrupt synaptobrevin II retrieval. J Neurosci. 2013;33(34):13695-13700. 